\title{
COMPARATIVE STUDY BETWEEN INTRAVENOUS KETAMINE OR MAGNESIUM SULFATE OR BOTH ON POSTOPERATIVE MORPHINE CONSUMPTION AFTER MAJOR ABDOMINAL SURGERY
}

\author{
By
Ibrahim Abd El-Raof Mohamed, Tarek Abd El-Salam Seleem and Abd El-Wahab Abd El-Sattar Saleh \\ Department of Anesthesiology and Intensive Care Faculty of Medicine, Al-Azhar \\ University \\ Corresponding author: Ebrahim A Raof Bashter,
}

Mobile: 01066867550, E-mail: ebrahimraof78@gmail.com

\begin{abstract}
Background: Intravenous patient-controlled analgesia (IV-PCA) with morphine is commonly used for postoperative pain control following major abdominal surgery. However, large amounts of morphine may lead to significant adverse events. Multimodal analgesia using a non-opioid analgesic, in addition to an opioid analgesic, has been suggested as a way to improve postoperative pain control and to reduce opioid use.

Objective: To compare the difference between intravenous Ketamine or Magnesium Sulfate or both on postoperative morphine consumption after major abdominal surgery.

Patients and Methods: This study included 100 patients of both sexes admitted for major abdominal surgery. They were randomly allocated into four equal groups. Group I: Control group, Group II received only ketamine, Group III received only magnesium sulfate and Group IV received both ketamine and magnesium sulfate. The following parameters were assessed in the four groups: Heart rate (HR), blood pressure (BP), respiratory rate (RR), end tidal $\mathrm{Co} 2$, the severity of pain on modified Ramsay sedation scale, time to first request for analgesia postoperatively, the number of rescue analgesia given, , adverse events, the level of the patient satisfaction and total dose of morphine consumption postoperatively (mg/48h).
\end{abstract}

Results: This study showed that the hemodynamics of the patients were more stable in (ketamine + magnesium sulfate) and ketamine only group than in magnesium sulfate only group and control group throughout all 48 hours postoperative. Usage of ketamine decreases postoperative pain and analgesic consumption in the first 48 hours after surgery along with longer pain free period compared to patients who were given magnesium sulfate only and control group. Ketamine is highly effective in postoperative pain control in major abdominal surgery without any hazards on patients. The total consumption of morphine, and additional analgesic requirements were less, while the satisfaction level of patients were higher in (ketamine+ magnesium sulfate) and ketamine only group.

Conclusion: Ketamine is one of the most advantageous adjuvant drugs for treating postoperative pain, while magnesium sulfate alone is less effective, with increase in opioid side effect. Combination of ketamine+ magnesium sulfate is better in treating postoperative pain and preserve patient hemodynamics with decrease in opioid side effect.

Key words: Ketamine, magnesium sulfate, postoperative morphine consumption, major abdominal surgery. 


\section{INTRODUCTION}

Opioid-based analgesia plays a significant role in the control of postsurgical pain; however, use of opioid may lead to significant side effects (e.g., nausea and vomiting) and adverse events (e.g., respiratory depression), which may be associated with significantly longer hospital stays and higher hospital costs in the postsurgical setting (Hurley and $W u$, 2010).

Since these adverse events occur more often in patients receiving higher doses of opioids, it is important to find ways to reduce opioid use in the postoperative period. Multimodal analgesia, using a non-opioid analgesic in addition to an opioid analgesic, has been suggested as a way to improve postoperative pain control and reduce opioid use (Ding et al., 2014).

Ketamine and Magnesium sulfate are non-opioid analgesics that have been studied as an adjuvant to opioid analgesics. They have been found to have anesthetic, analgesic, and they has been suggested that they may play a role in reducing analgesic requirements during the postoperative period (Albrecht et al., 2013).

Effective postoperative pain control is an essential component of the care of the surgical patient. Inadequate pain control, apart from being inhumane, may result in increased morbidity or mortality. Evidence suggests that surgery suppresses the immune system and that this suppression is proportionate to the invasiveness of the surgery. Good analgesia can reduce this deleterious effect (Ding et al., 2014).
The aim of this study was to compare the difference between intravenous Ketamine and or Magnesium Sulfate on postoperative morphine consumption after major abdominal surgery as primary outcome and to measure the hemodynamic effects and intensity of pain as secondary outcome.

\section{PATIENTS AND METHODS}

After approval of the medical ethical committee at Al-Azhar University Hospitals, department of anesthesiology, 100 patients at the Department of Anesthesiology and Intensive Care were scheduled according to the American Society of Anesthesiologist (ASA) physical status I or II, of either sex, age (30-60) years for major abdominal operation under general anesthesia are enrolled in this prospective controlled double blinded randomized study.

Information about the study was given comprehensively both orally and in written form to the patients. All patients gave their written informed consents prior to their inclusion in the study.

\section{Exclusion criteria:}

All patients with cardiovascular, pulmonary, psychological or neurological diseases, patients known to be epileptic, patients who have an increase in intracranial tension, patients with known allergies to the study drug (ketaminemagnesium sulfate), patients with recent NSAID medication, history of current regular use of analgesics, anticonvulsants, antidepressants, or opioids within the last month, patients with any perioperative complications, physical status: ASA III or above, pregnant ladies, body mass index more than 35, coagulation disorder, 
history of chronic pain and patients with drug or alcohol abuse, were excluded from the study.

\section{Patients are randomized into the four groups:}

All patients received morphine at infusion rate of $0.25 \mathrm{mg} / \mathrm{h}$, and received the studied drugs in the four groups diluted in $50 \mathrm{cc}$ syringe containing a normal saline $0.9 \%$ with infusion rate 1 $\mathrm{cm} / \mathrm{h}$.

Control group (Group I): received a bolus dose, and continuous intravenous infusion of normal saline.

Ketamine group (Group II): received a bolus dose of ketamine $(0.2 \mathrm{mg} / \mathrm{kg})$ intravenously, followed by continuous infusion of ketamine $(0.05 \mathrm{mg} / \mathrm{kg} / \mathrm{h})$.

Magnesium group (Group III): received a bolus dose of magnesium sulphate $(50 \mathrm{mg} / \mathrm{kg})$ intravenously, followed by continuous infusion of magnesium $(10 \mathrm{mg} / \mathrm{kg} / \mathrm{h})$.

Ketamine and Magnesium Sulphate group (Group IV): received a bolus dose of ketamine $(0.1 \mathrm{mg} / \mathrm{kg})$, and magnesium sulphate $(25 \mathrm{mg} / \mathrm{kg})$ intravenously, then continuous infusion of $(0.025 \mathrm{mg} / \mathrm{kg} / \mathrm{h})$ ketamine $+(25$ $\mathrm{mg} / \mathrm{kg} / \mathrm{h}$ ) magnesium sulphate.

Rescue analgesia: $5 \mathrm{mg}$ morphine IV, followed by pain assessment after 15 minutes if the pain score $>4$ piroxicam 20 $\mathrm{mg}$ was given intravenously.

All patients were screened for suitability by history including assessment of cardio respiratory status, physical examination for heart, chest. Investigations for $\mathrm{CBC}$, coagulation profile, liver function, kidney function and
ECG or specific investigations according to the patients.

Patient monitoring (standard monitoring) by pulse oximetry, ECG, Non-invasive blood pressure monitoring, $5 \mathrm{~min}$. interval and capnpgram.

Pre oxygenation with $100 \%$ oxygen was done for $3 \mathrm{~min}$. General anesthesia was induced with an injection of Fentanyl (2 $\mu \mathrm{g} / \mathrm{kg})$, Propofol (1-2 mg/kg) followed by Atracurium $(0.5 \mathrm{mg} / \mathrm{kg})$ to facilitate orotracheal intubation. Anesthesia was maintained using isoflurane in an air/oxygen mixture. Intermittent boluses of Atracurium were given to achieve muscle relaxation. Minute ventilation was adjusted to maintain normocapnia (end tidal carbon dioxide; etCO2, between 34 and $38 \mathrm{~mm} \mathrm{Hg}$ ). Ten minutes before the end of surgery, morphine $(0.05 \mathrm{mg} / \mathrm{kg})$ was routinely administered IV.

At the end of surgery, anesthesia discontinued, and residual neuromuscular blockade was antagonized with neostigmine $(0.08 \mathrm{mg} / \mathrm{kg})$ and atropine $(0.02 \mathrm{mg} / \mathrm{kg})$.followed by extubation. When the patients became fully awake; patients transferred to the postanesthesia care unit (PACU). The bolus doses of the study drugs were administered, and their infusions were started simultaneously with the initiation of the IV-PCA morphine. All studied solutions were continued until 48 $\mathrm{h}$ postoperatively via an infuser. Thus, patients have two separate mechanical infusion devices during the study period.

Heart rate, blood pressure, oxygen saturation, respiratory rate, and end-tidal $\mathrm{PaCO}_{2}$. The severity of pain (with NPRS) were recorded at $0,30 \mathrm{~min}$, 1 hour, $2 \mathrm{~h}, 4 \mathrm{~h}$, $6 \mathrm{~h}, 8 \mathrm{~h}, 10 \mathrm{~h}, 12 \mathrm{~h}, 18 \mathrm{~h}, 24 \mathrm{~h}$, and every 12 hours to the end point of the study period. 
The degree of sedation was assessed using modified Ramsay sedation scale, Time of first request for analgesia postoperatively (hours), Number of rescue analgesic given, Total dose of morphine postoperatively (mg/48h). Adverse events such as nausea, vomiting, pruritus, hypotension, bradycardia and the level of the patient satisfaction will be recorded according to four level satisfaction score: Very satisfied (4), generally satisfied (3), moderately satisfied (2), and unsatisfied (1).

Recorded data were analyzed using the statistical package for the social sciences, version. 20.0 (SPSS Inc., Chicago, Illinois, USA). Quantitative data were expressed as mean \pm standard deviation (SD). Qualitative data were expressed as frequency and percentage. One way ANOVA test was used to compare means and Fisher exact test was used to compare proportions between qualitative parameters. The confidence interval was set to $95 \%$ and the margin of error accepted was set to $5 \%$.

P-value $\quad<0.05$ was considered significant.

\section{RESULTS}

As regard demographic data among the four groups, there were no statistical differences among them (Table 1).

Table (1): Comparison among groups according to demographic data

\begin{tabular}{|c|c|c|c|c|c|c|}
\hline \multirow{2}{*}{ Parameters Groups } & Group I & Group II & Group III & Group IV & \multirow{2}{*}{ P-value } \\
\cline { 2 - 6 } & No. $=\mathbf{2 5}$ & No. $=\mathbf{2 5}$ & No. = 25 & No. = 25 & \\
\hline \multirow{2}{*}{ Gender } & Male & $13(52.0 \%)$ & $14(56.0 \%)$ & $14(56.0 \%)$ & $12(48.0 \%)$ & \multirow{2}{*}{0.931} \\
\cline { 2 - 6 } & Female & $12(48.0 \%)$ & $11(44.0 \%)$ & $11(44.0 \%)$ & $13(52.0 \%)$ & \\
\hline \multirow{2}{*}{ Age } & Mean \pm SD & $43.15 \pm 8.53$ & $42.20 \pm 8.68$ & $44.00 \pm 9.04$ & $41.52 \pm 8.78$ & \multirow{2}{*}{0.765} \\
\cline { 2 - 6 } & Range & $30-60$ & $30-58$ & $30-59$ & $30-59$ & \\
\hline
\end{tabular}

There was statistically highly significant difference between groups in post-operative heart rate at $1 \mathrm{hr}, 2 \mathrm{hr}, 4 \mathrm{hr}$, $6 \mathrm{hr}, 8 \mathrm{hr}, 10 \mathrm{hr}, 12 \mathrm{hr}, 18 \mathrm{~h}$ and $24 \mathrm{hr}$, and non- significant difference between groups at $30 \mathrm{~min}, 36 \mathrm{hr}$ and $48 \mathrm{hrfrom}$ postoperative care unit (PACU) till the end of the first 48 hours after the operation (Table 2). 
Table (2): Comparison between groups according to postoperative heart rate (beat/min-Mean \pm SD)

\begin{tabular}{|c|c|c|c|c|c|}
\hline $\begin{array}{l}\text { Heart rate } \\
\text { (beat/min) }\end{array}$ & Group I & Group II & Group III & Group IV & P-value \\
\hline $30 \mathrm{~min}$ & $115.00 \pm 14.38$ & $110.00 \pm 13.75$ & $112.00 \pm 14.00$ & $108.00 \pm 13.50$ & 0.332 \\
\hline $1 \mathrm{hr}$ & $105.00 \pm 13.13$ & $88.00 \pm 11.00$ & $100.00 \pm 12.50$ & $85.00 \pm 10.63$ & $<0.001$ \\
\hline $2 \mathrm{hr}$ & $90.00 \pm 11.25$ & $70.00 \pm 8.75$ & $95.00 \pm 11.88$ & $68.00 \pm 8.50$ & $<0.001$ \\
\hline $4 \mathrm{hr}$ & $85.00 \pm 10.63$ & $75.00 \pm 9.38$ & $90.00 \pm 11.25$ & $70.00 \pm 8.75$ & $<0.001$ \\
\hline $6 \mathrm{hr}$ & $100.00 \pm 12.50$ & $66.00 \pm 8.25$ & $90.00 \pm 11.25$ & $66.00 \pm 8.25$ & $<0.001$ \\
\hline $8 \mathrm{hr}$ & $102.00 \pm 12.75$ & $80.00 \pm 10.00$ & $91.00 \pm 11.38$ & $79.00 \pm 9.88$ & $<0.001$ \\
\hline $10 \mathrm{hr}$ & $90.00 \pm 11.25$ & $81.00 \pm 10.13$ & $85.00 \pm 10.63$ & $78.00 \pm 9.75$ & $<0.001$ \\
\hline $12 \mathrm{hr}$ & $85.00 \pm 10.63$ & $75.00 \pm 9.38$ & $84.00 \pm 10.50$ & $72.00 \pm 9.00$ & $<0.001$ \\
\hline $18 \mathrm{hr}$ & $89.00 \pm 11.13$ & $70.00 \pm 8.75$ & $87.00 \pm 10.88$ & $70.00 \pm 8.75$ & $<0.001$ \\
\hline $24 \mathrm{hr}$ & $80.00 \pm 10.00$ & $68.00 \pm 8.50$ & $80.00 \pm 10.00$ & $65.00 \pm 8.13$ & $<0.001$ \\
\hline $36 \mathrm{hr}$ & $75.00 \pm 9.38$ & $70.00 \pm 8.75$ & $70.00 \pm 8.75$ & $70.00 \pm 8.75$ & 0.124 \\
\hline $48 \mathrm{hr}$ & $75.00 \pm 9.38$ & $73.00 \pm 9.13$ & $73.00 \pm 9.13$ & $71.00 \pm 8.88$ & 0.497 \\
\hline
\end{tabular}

There was a statistically significant highly difference between groups in postoperative systolic blood pressure at $2 \mathrm{hr}$, $10 \mathrm{hr}, 12 \mathrm{hr}, 18 \mathrm{hr}$ and $48 \mathrm{hr}$, and nonsignificant difference between groups at
$30 \mathrm{~min}, 1 \mathrm{hr}, 4 \mathrm{hr}, 6 \mathrm{hr}, 8 \mathrm{hr}, 24 \mathrm{hr}$ and $36 \mathrm{hr}$ from postoperative care unit (PACU) till the end of the first 48 hours after the operation (Table 3).

Table (3): Comparison between groups according to postoperative systolic blood pressure $(\mathbf{m m H g}$ - Mean \pm SD)

\begin{tabular}{|c|c|c|c|c|c|}
\hline${ }_{\text {SBP }}^{\text {Groups }}$ & Group I & Group II & Group III & Group IV & P-value \\
\hline $30 \mathrm{~min}$ & $150.00 \pm 18.75$ & $145.00 \pm 18.13$ & $153.00 \pm 19.13$ & $140.00 \pm 17.50$ & 0.071 \\
\hline $1 \mathrm{hr}$ & $143.00 \pm 16.88$ & $135.00 \pm 16.25$ & $137.00 \pm 16.88$ & $130.00 \pm 16.25$ & 0.054 \\
\hline $2 \mathrm{hr}$ & $140.00 \pm 17.50$ & $125.00 \pm 15.63$ & $130.00 \pm 16.25$ & $122.00 \pm 15.25$ & $<0.001$ \\
\hline $4 \mathrm{hr}$ & $130.00 \pm 16.25$ & $121.00 \pm 15.13$ & $126.00 \pm 15.75$ & $120.00 \pm 15.00$ & 0.089 \\
\hline $6 \mathrm{hr}$ & $125.00 \pm 15.63$ & $118.00 \pm 14.75$ & $123.00 \pm 15.38$ & $115.00 \pm 14.38$ & 0.081 \\
\hline $8 \mathrm{hr}$ & $130.00 \pm 16.25$ & $125.00 \pm 15.63$ & $129.00 \pm 16.13$ & $125.00 \pm 15.63$ & 0.565 \\
\hline $10 \mathrm{hr}$ & $135.00 \pm 16.88$ & $127.00 \pm 15.88$ & $130.00 \pm 16.25$ & $110.00 \pm 13.75$ & $<0.001$ \\
\hline $12 \mathrm{hr}$ & $134.00 \pm 16.75$ & $113.00 \pm 14.13$ & $129.00 \pm 16.13$ & $115.00 \pm 14.38$ & $<0.001$ \\
\hline $18 \mathrm{hr}$ & $143.00 \pm 17.88$ & $125.00 \pm 15.63$ & $133.00 \pm 16.63$ & $119.00 \pm 14.88$ & $<0.001$ \\
\hline $24 \mathrm{hr}$ & $133.00 \pm 16.38$ & $129.00 \pm 16.13$ & $130.00 \pm 16.13$ & $125.00 \pm 15.63$ & 0.0371 \\
\hline $36 \mathrm{hr}$ & $125.00 \pm 15.63$ & $121.00 \pm 15.13$ & $122.00 \pm 15.25$ & $118.00 \pm 14.75$ & 0.443 \\
\hline $48 \mathrm{hr}$ & $129.00 \pm 16.13$ & $115.00 \pm 14.38$ & $125.00 \pm 15.63$ & $115.00 \pm 14.38$ & 0.002 \\
\hline
\end{tabular}

There was a statistically highly significant difference between groups in post-operative diastolic blood pressure at $1 \mathrm{hr} 2 \mathrm{hr}, 4 \mathrm{hr}, 6 \mathrm{hr}, 10 \mathrm{hr}, 12 \mathrm{hr}, 12 \mathrm{hr}, 18 \mathrm{hr}$, $24 \mathrm{hr}, 36 \mathrm{hr}$ and $48 \mathrm{hr}$ and non-significant difference between groups at $30 \mathrm{~min}$ and $8 \mathrm{hr}$ from postoperative care unit (PACU) till the end of the first 48 hours after the operation (Table 4). 
Table (4): Comparison between groups according to postoperative diastolic blood pressure $(\mathrm{mmHg}-$ Mean $\pm \mathrm{SD})$

\begin{tabular}{|c|c|c|c|c|c|}
\hline DBP Groups & Group I & Group II & Group III & Group IV & P-value \\
\hline $30 \mathrm{~min}$ & $105.00 \pm 13.13$ & $100.00 \pm 12.50$ & $101.00 \pm 12.63$ & $100.00 \pm 12.50$ & 0.455 \\
\hline $1 \mathrm{hr}$ & $100.00 \pm 12.50$ & $90.00 \pm 11.25$ & $91.00 \pm 11.38$ & $90.00 \pm 11.25$ & 0.006 \\
\hline $2 \mathrm{hr}$ & $95.00 \pm 11.88$ & $85.00 \pm 10.63$ & $90.00 \pm 11.25$ & $84.00 \pm 10.50$ & 0.002 \\
\hline $4 \mathrm{hr}$ & $93.00 \pm 11.63$ & $78.00 \pm 9.75$ & $88.00 \pm 11.00$ & $77.00 \pm 9.63$ & $<0.001$ \\
\hline $6 \mathrm{hr}$ & $97.00 \pm 12.13$ & $81.00 \pm 10.13$ & $85.00 \pm 10.63$ & $80.00 \pm 10.00$ & $<0.001$ \\
\hline $8 \mathrm{hr}$ & $90.00 \pm 11.25$ & $86.00 \pm 10.75$ & $90.00 \pm 11.25$ & $85.00 \pm 10.63$ & 0.237 \\
\hline $10 \mathrm{hr}$ & $95.00 \pm 11.88$ & $89.00 \pm 11.13$ & $95.00 \pm 11.88$ & $70.00 \pm 8.75$ & $<0.001$ \\
\hline $12 \mathrm{hr}$ & $93.00 \pm 11.63$ & $80.00 \pm 10.00$ & $91.00 \pm 11.38$ & $73.00 \pm 9.13$ & $<0.001$ \\
\hline $18 \mathrm{hr}$ & $99.00 \pm 12.38$ & $85.00 \pm 10.63$ & $88.00 \pm 11.00$ & $79.00 \pm 9.88$ & $<0.001$ \\
\hline $24 \mathrm{hr}$ & $98.00 \pm 12.25$ & $83.00 \pm 10.38$ & $95.00 \pm 11.88$ & $80.00 \pm 10.00$ & $<0.001$ \\
\hline $36 \mathrm{hr}$ & $85.00 \pm 10.63$ & $80.00 \pm 10.00$ & $84.00 \pm 10.50$ & $73.00 \pm 9.13$ & $<0.001$ \\
\hline $48 \mathrm{hr}$ & $88.00 \pm 11.00$ & $79.00 \pm 9.88$ & $81.00 \pm 10.13$ & $70.00 \pm 8.75$ & $<0.001$ \\
\hline
\end{tabular}

A statistically highly significant difference was found between groups in post-operative $\mathrm{O} 2$ saturation at $6 \mathrm{hr}, 8 \mathrm{hr}$, $10 \mathrm{hr}$ and $18 \mathrm{hr}$, and significant difference between groups at $2 \mathrm{hr}$, and non- significant difference between groups at $30 \mathrm{~min}$, $1 \mathrm{hr}, 4 \mathrm{hr}, 12 \mathrm{hr}, 24 \mathrm{hr}, \quad 36 \mathrm{hr}$ and 48 from postoperative care unit (PACU) till the end of the first 48 hours after the operation (Table 5).

Table (5): Comparison between groups according to postoperative 02 saturation. $($ Mean \pm SD)

\begin{tabular}{|c|c|c|c|c|c|}
\hline O2 Saturation & \multirow{2}{*}{ Group I } & Group II & Group III & Group IV & P-value \\
\hline $30 \mathrm{~min}$ & $95.00 \pm 2.30$ & $97.00 \pm 1.30$ & $96.00 \pm 2.30$ & $97.00 \pm 3.50$ & 0.284 \\
\hline $1 \mathrm{hr}$ & $97.00 \pm 2.50$ & $99.00 \pm 3.50$ & $99.00 \pm 3.60$ & $99.00 \pm 3.50$ & 0.101 \\
\hline $2 \mathrm{hr}$ & $96.00 \pm 3.50$ & $99.00 \pm 3.60$ & $98.00 \pm 2.65$ & $100.00 \pm 4.35$ & 0.01 \\
\hline $4 \mathrm{hr}$ & $97.00 \pm 3.80$ & $98.00 \pm 4.50$ & $97.00 \pm 3.33$ & $98.00 \pm 3.58$ & 0.758 \\
\hline $6 \mathrm{hr}$ & $95.00 \pm 1.20$ & $99.00 \pm 2.80$ & $96.00 \pm 3.59$ & $99.00 \pm 4.25$ & 0.001 \\
\hline $8 \mathrm{hr}$ & $95.00 \pm 2.98$ & $98.00 \pm 2.36$ & $97.00 \pm 2.87$ & $98.00 \pm 3.58$ & 0.002 \\
\hline $10 \mathrm{hr}$ & $93.00 \pm 1.25$ & $98.00 \pm 2.78$ & $97.00 \pm 2.25$ & $99.00 \pm 5.65$ & $<0.001$ \\
\hline $12 \mathrm{hr}$ & $98.00 \pm 1.23$ & $98.00 \pm 4.35$ & $98.00 \pm 3.58$ & $100.00 \pm 4.00$ & 0.115 \\
\hline $18 \mathrm{hr}$ & $96.00 \pm 2.89$ & $99.00 \pm 4.58$ & $99.00 \pm 3.25$ & $100.00 \pm 4.25$ & 0.002 \\
\hline $24 \mathrm{hr}$ & $97.00 \pm 3.58$ & $99.00 \pm 3.65$ & $99.00 \pm 2.99$ & $100.00 \pm 4.22$ & 0.108 \\
\hline $36 \mathrm{hr}$ & $97.00 \pm 3.41$ & $99.00 \pm 3.74$ & $98.00 \pm 3.89$ & $99.00 \pm 2.30$ & 0.274 \\
\hline $48 \mathrm{hr}$ & $99.00 \pm 2.15$ & $99.00 \pm 2.98$ & $99.00 \pm 4.02$ & $99.00 \pm 3.58$ & 1 \\
\hline
\end{tabular}

There was a statistically significant highly difference between groups in postoperative respiratory rate at $30 \mathrm{~min}, 1 \mathrm{hr}$, $2 \mathrm{hr}, 6 \mathrm{hr}, 8 \mathrm{hr}, 10 \mathrm{hr}, 12 \mathrm{hr}$ and $24 \mathrm{hr}$,and nonsignificant difference between groups at 4hr, 18hr, $36 \mathrm{hr}$ and $48 \mathrm{hr}$ from postoperative care unit (PACU) till the end of the first 48 hours after the operation (Table 6). 
Table (6): Comparison between groups according to postoperative respiratory rate $($ RR-Mean \pm SD)

\begin{tabular}{|c|c|c|c|c|c|}
\hline RR Groups & Group I & Group II & Group III & Group IV & P-value \\
\hline $30 \mathrm{~min}$ & $24.00 \pm 3.00$ & $21.00 \pm 2.63$ & $23.00 \pm 2.88$ & $20.00 \pm 2.50$ & $<0.001$ \\
\hline $1 \mathrm{hr}$ & $20.00 \pm 2.50$ & $17.00 \pm 2.13$ & $18.00 \pm 2.25$ & $16.00 \pm 2.00$ & $<0.001$ \\
\hline $2 \mathrm{hr}$ & $17.00 \pm 2.13$ & $15.00 \pm 1.88$ & $17.00 \pm 2.13$ & $15.00 \pm 1.88$ & $<0.001$ \\
\hline $4 \mathrm{hr}$ & $16.00 \pm 2.00$ & $15.00 \pm 1.88$ & $16.00 \pm 2.00$ & $15.00 \pm 1.88$ & 0.092 \\
\hline $6 \mathrm{hr}$ & $17.00 \pm 2.13$ & $16.00 \pm 2.00$ & $15.00 \pm 1.88$ & $14.00 \pm 1.75$ & $<0.001$ \\
\hline $8 \mathrm{hr}$ & $18.00 \pm 2.38$ & $15.00 \pm 1.88$ & $17.00 \pm 2.13$ & $13.00 \pm 1.63$ & $<0.001$ \\
\hline $10 \mathrm{hr}$ & $17.00 \pm 2.63$ & $14.00 \pm 1.75$ & $16.00 \pm 2.00$ & $14.00 \pm 1.75$ & $<0.001$ \\
\hline $12 \mathrm{hr}$ & $15.00 \pm 1.88$ & $14.00 \pm 1.75$ & $14.00 \pm 1.75$ & $13.00 \pm 1.63$ & 0.002 \\
\hline $18 \mathrm{hr}$ & $14.00 \pm 1.75$ & $13.00 \pm 1.63$ & $13.00 \pm 1.63$ & $13.00 \pm 1.63$ & 0.086 \\
\hline $24 \mathrm{hr}$ & $15.00 \pm 1.88$ & $14.00 \pm 1.75$ & $14.00 \pm 1.75$ & $13.00 \pm 1.63$ & 0.002 \\
\hline $36 \mathrm{hr}$ & $14.00 \pm 1.75$ & $14.00 \pm 1.75$ & $14.00 \pm 1.75$ & $13.00 \pm 1.63$ & 0.104 \\
\hline $48 \mathrm{hr}$ & $14.00 \pm 1.75$ & $14.00 \pm 1.75$ & $14.00 \pm 1.75$ & $13.00 \pm 1.63$ & 0.104 \\
\hline
\end{tabular}

There was a statistically significant highly difference between groups in postoperative end tidal $\mathrm{CO} 2$ at $2 \mathrm{hr}, 8 \mathrm{hr}, 10 \mathrm{hr}$, $12 \mathrm{hr}$ and $18 \mathrm{hr}$,and significant difference between groups at $24 \mathrm{hr}, 36 \mathrm{hr}, 48 \mathrm{hr}$. and non-significant difference between groups at $30 \mathrm{~min}, 1 \mathrm{hr}, 4 \mathrm{hr}$ and $6 \mathrm{hr}$ from postoperative care unit (PACU) till the end of the first 48 hours after the operation (Table 7).

Table (7): Comparison between groups according to postoperative End tidal $\mathrm{CO} 2$ $($ Mean \pm SD)

\begin{tabular}{|c|c|c|c|c|c|}
\hline $\begin{array}{ll}\text { End Tidal } \mathrm{CO}_{2} & \text { Groups } \\
\end{array}$ & Group I & Group II & Group III & Group IV & P-value \\
\hline $30 \mathrm{~min}$ & $31.00 \pm 3.88$ & $30.00 \pm 3.75$ & $31.00 \pm 3.88$ & $29.00 \pm 3.63$ & 0.195 \\
\hline $1 \mathrm{hr}$ & $29.00 \pm 3.63$ & $28.00 \pm 3.50$ & $29.00 \pm 3.63$ & $27.00 \pm 3.38$ & 0.146 \\
\hline $2 \mathrm{hr}$ & $35.00 \pm 4.38$ & $31.00 \pm 3.88$ & $33.00 \pm 4.13$ & $29.00 \pm 3.63$ & $<0.001$ \\
\hline $4 \mathrm{hr}$ & $32.00 \pm 4.00$ & $31.00 \pm 3.88$ & $31.00 \pm 3.88$ & $30.00 \pm 3.75$ & 0.350 \\
\hline $6 \mathrm{hr}$ & $35.00 \pm 4.38$ & $34.00 \pm 4.25$ & $35.00 \pm 4.38$ & $34.00 \pm 4.25$ & 0.720 \\
\hline $8 \mathrm{hr}$ & $39.00 \pm 4.88$ & $31.00 \pm 3.88$ & $37.00 \pm 4.63$ & $29.00 \pm 3.63$ & $<0.001$ \\
\hline $10 \mathrm{hr}$ & $38.00 \pm 4.75$ & $35.00 \pm 3.50$ & $38.00 \pm 4.75$ & $34.00 \pm 3.13$ & $<0.001$ \\
\hline $12 \mathrm{hr}$ & $39.00 \pm 4.88$ & $31.00 \pm 3.88$ & $39.00 \pm 4.88$ & $30.00 \pm 3.75$ & $<0.001$ \\
\hline $18 \mathrm{hr}$ & $38.00 \pm 4.75$ & $35.00 \pm 4.38$ & $37.00 \pm 4.63$ & $34.00 \pm 4.25$ & 0.009 \\
\hline $24 \mathrm{hr}$ & $40.00 \pm 5.00$ & $37.00 \pm 4.63$ & $38.00 \pm 4.75$ & $36.00 \pm 4.50$ & 0.025 \\
\hline $36 \mathrm{hr}$ & $40.00 \pm 5.00$ & $38.00 \pm 4.75$ & $39.00 \pm 4.88$ & $36.00 \pm 4.50$ & 0.027 \\
\hline $48 \mathrm{hr}$ & $39.00 \pm 4.88$ & $38.00 \pm 4.75$ & $39.00 \pm 4.88$ & $35.00 \pm 4.38$ & 0.010 \\
\hline
\end{tabular}

A statistically significant highly difference was received between groups in post-operative Ramsay sedation scale at $2 \mathrm{hr}, 8 \mathrm{hr}, 10 \mathrm{hr}, 12 \mathrm{hr}, 18 \mathrm{hr}, 24 \mathrm{hr}, 36 \mathrm{hr}$ and $48 \mathrm{hr}$,and non-significant difference between groups at $30 \mathrm{~min}, 1 \mathrm{hr}, 4 \mathrm{hr}$ and $6 \mathrm{hr}$ from postoperative care unit (PACU) till the end of the first 48 hours after the operation (Table 8). 
Table (8): Comparison between groups according to postoperative Ramsay Sedation Scale $($ Mean \pm SD)

\begin{tabular}{|c|c|c|c|c|c|}
\hline $\begin{array}{l}\text { Ramsay } \\
\text { Sedation Scale }\end{array}$ & Group I & Group II & Group III & Group IV & P-value \\
\hline $30 \mathrm{~min}$ & $1.00 \pm 0.13$ & $1.00 \pm 0.13$ & $1.00 \pm 0.13$ & $1.00 \pm 0.13$ & 1 \\
\hline $1 \mathrm{hr}$ & $1.00 \pm 0.13$ & $1.00 \pm 0.13$ & $1.00 \pm 0.13$ & $1.00 \pm 0.13$ & 1 \\
\hline $2 \mathrm{hr}$ & $2.00 \pm 0.25$ & $1.00 \pm 0.13$ & $2.00 \pm 0.25$ & $1.00 \pm 0.13$ & $<0.001$ \\
\hline $4 \mathrm{hr}$ & $2.00 \pm 0.25$ & $2.00 \pm 0.25$ & $2.00 \pm 0.25$ & $2.00 \pm 0.25$ & 1 \\
\hline $6 \mathrm{hr}$ & $2.00 \pm 0.25$ & $2.00 \pm 0.25$ & $2.00 \pm 0.25$ & $2.00 \pm 0.25$ & 1 \\
\hline $8 \mathrm{hr}$ & $3.00 \pm 0.38$ & $2.00 \pm 0.25$ & $2.00 \pm 0.25$ & $1.00 \pm 0.13$ & $<0.001$ \\
\hline $10 \mathrm{hr}$ & $3.00 \pm 0.38$ & $1.00 \pm 0.13$ & $3.00 \pm 0.38$ & $1.00 \pm 0.13$ & $<0.001$ \\
\hline $12 \mathrm{hr}$ & $3.00 \pm 0.38$ & $2.00 \pm 0.25$ & $3.00 \pm 0.38$ & $1.00 \pm 0.13$ & $<0.001$ \\
\hline $18 \mathrm{hr}$ & $3.00 \pm 0.38$ & $2.00 \pm 0.25$ & $2.00 \pm 0.25$ & $2.00 \pm 0.25$ & $<0.001$ \\
\hline $24 \mathrm{hr}$ & $2.00 \pm 0.25$ & $2.00 \pm 0.25$ & $2.00 \pm 0.25$ & $1.00 \pm 0.13$ & $<0.001$ \\
\hline $36 \mathrm{hr}$ & $2.00 \pm 0.25$ & $2.00 \pm 0.25$ & $2.00 \pm 0.25$ & $1.00 \pm 0.13$ & $<0.001$ \\
\hline $48 \mathrm{hr}$ & $2.00 \pm 0.25$ & $2.00 \pm 0.25$ & $2.00 \pm 0.25$ & $1.00 \pm 0.13$ & $<0.001$ \\
\hline
\end{tabular}

Statistically highly significant difference occurred between groups in post-operative rescue analgesia given at $6 \mathrm{hr}$, and significant difference between groups at $1 \mathrm{hr}, 4 \mathrm{hr}$ and $12 \mathrm{hr}$,and non- significant difference between groups at $2 \mathrm{hr}, 10 \mathrm{hr}$, and $36 \mathrm{hr}$ from postoperative care unit (PACU) till the end of the first 48 hours after the operation (Table 9).

Table (9): Comparison between groups according to postoperative rescue analgesia given $($ Mean $\pm \mathrm{SD})$

\begin{tabular}{|c|c|c|c|c|c|c|c|c|c|}
\hline \multirow{2}{*}{$\begin{array}{l}\text { Rescue Groups } \\
\text { analgesia given }\end{array}$} & \multicolumn{2}{|c|}{ Group I } & \multicolumn{2}{|c|}{ Group II } & \multicolumn{2}{c|}{ Group III } & \multicolumn{2}{c|}{ Group IV } & \multirow{2}{*}{ P-value } \\
\cline { 2 - 11 } & No. & $\%$ & No. & $\%$ & No. & $\%$ & No. & $\%$ & \\
\hline $30 \mathrm{~min}$ & 0 & $0.0 \%$ & 0 & $0.0 \%$ & 0 & $0.0 \%$ & 0 & $0.0 \%$ & - \\
\hline $1 \mathrm{hr}$ & 4 & $16.0 \%$ & 0 & $0.0 \%$ & 1 & $4.0 \%$ & 0 & $0.0 \%$ & 0.029 \\
\hline $2 \mathrm{hr}$ & 2 & $8.0 \%$ & 0 & $0.0 \%$ & 1 & $4.0 \%$ & 0 & $0.0 \%$ & 0.286 \\
\hline $4 \mathrm{hr}$ & 6 & $24.0 \%$ & 2 & $8.0 \%$ & 5 & $20.0 \%$ & 0 & $0.0 \%$ & 0.045 \\
\hline $6 \mathrm{hr}$ & 8 & $32.0 \%$ & 0 & $0.0 \%$ & 4 & $16.0 \%$ & 0 & $0.0 \%$ & $<0.001$ \\
\hline $8 \mathrm{hr}$ & 0 & $0.0 \%$ & 0 & $0.0 \%$ & 0 & $0.0 \%$ & 0 & $0.0 \%$ & - \\
\hline $10 \mathrm{hr}$ & 3 & $12.0 \%$ & 2 & $8.0 \%$ & 3 & $12.0 \%$ & 1 & $4.0 \%$ & 0.719 \\
\hline $12 \mathrm{hr}$ & 3 & $12.0 \%$ & 0 & $0.0 \%$ & 0 & $0.0 \%$ & 0 & $0.0 \%$ & 0.026 \\
\hline $18 \mathrm{hr}$ & 0 & $0.0 \%$ & 0 & $0.0 \%$ & 0 & $0.0 \%$ & 0 & $0.0 \%$ & - \\
\hline $24 \mathrm{hr}$ & 0 & $0.0 \%$ & 0 & $0.0 \%$ & 0 & $0.0 \%$ & 0 & $0.0 \%$ & - \\
\hline $36 \mathrm{hr}$ & 1 & $4.0 \%$ & 0 & $0.0 \%$ & 1 & $4.0 \%$ & 0 & $0.0 \%$ & 0.564 \\
\hline $48 \mathrm{hr}$ & 0 & $0.0 \%$ & 0 & $0.0 \%$ & 0 & $0.0 \%$ & 0 & $0.0 \%$ & - \\
\hline
\end{tabular}

Statistically highly significant difference occurred between groups in post-operative total morphine consumption at the end of the first 48 hours after the operation (Table 10). 
Table (10): Comparison between groups according to postoperative total morphine consumption (Mean \pm SD)

\begin{tabular}{|c|c|c|c|c|c|}
\hline $\begin{array}{l}\text { Total Groups } \\
\text { Morphine Consumption }\end{array}$ & Group I & Group II & Group III & Group IV & P-value \\
\hline $48 \mathrm{hr}$ & $25.32 \pm 4.37$ & $17.27 \pm 2.80$ & $23.84 \pm 4.75$ & $15.22 \pm 3.40$ & $<0.001$ \\
\hline
\end{tabular}

Statistically significant highly difference occurred between groups in post-operative nausea and vomiting at $10 \mathrm{hr}$ and $12 \mathrm{hr}$, and significant difference between groups at $8 \mathrm{hr}$, and non-significant difference between groups at $48 \mathrm{hr}$ from postoperative care unit (PACU) till the end of the first 48 hours after the operation (Table 11).

Table (11): Comparison between groups according to postoperative Nausea and vomiting (Mean \pm SD)

\begin{tabular}{|c|c|c|c|c|c|c|c|c|c|}
\hline \multirow{2}{*}{\begin{tabular}{ll|}
$\begin{array}{l}\text { Nausea } \\
+ \text { Vomiting }\end{array}$ & Groups \\
\end{tabular}} & \multicolumn{2}{|c|}{ Group I } & \multicolumn{2}{|c|}{ Group II } & \multicolumn{2}{|c|}{ Group III } & \multicolumn{2}{|c|}{ Group IV } & \multirow{2}{*}{$\begin{array}{c}P \text { - } \\
\text { value }\end{array}$} \\
\hline & No. & $\%$ & No. & $\%$ & No. & $\%$ & No. & $\%$ & \\
\hline $30 \mathrm{~min}$ & 5 & $20.0 \%$ & 3 & $12.0 \%$ & 4 & $16.0 \%$ & 1 & $4.0 \%$ & 0.377 \\
\hline $1 \mathrm{hr}$ & 0 & $0.0 \%$ & 0 & $0.0 \%$ & 0 & $0.0 \%$ & 0 & $0.0 \%$ & - \\
\hline $2 \mathrm{hr}$ & 0 & $0.0 \%$ & 0 & $0.0 \%$ & 0 & $0.0 \%$ & 0 & $0.0 \%$ & - \\
\hline $4 \mathrm{hr}$ & 0 & $0.0 \%$ & 0 & $0.0 \%$ & 0 & $0.0 \%$ & 0 & $0.0 \%$ & - \\
\hline $6 \mathrm{hr}$ & 0 & $0.0 \%$ & 0 & $0.0 \%$ & 0 & $0.0 \%$ & 0 & $0.0 \%$ & - \\
\hline $8 \mathrm{hr}$ & 7 & $28.0 \%$ & 3 & $12.0 \%$ & 2 & $8.0 \%$ & 0 & $0.0 \%$ & 0.02 \\
\hline $10 \mathrm{hr}$ & 3 & $12.0 \%$ & 0 & $0.0 \%$ & 1 & $4.0 \%$ & 9 & $36.0 \%$ & $<0.001$ \\
\hline $12 \mathrm{hr}$ & 1 & $4.0 \%$ & 0 & $0.0 \%$ & 8 & $32.0 \%$ & 0 & $0.0 \%$ & $<0.001$ \\
\hline $18 \mathrm{hr}$ & 0 & $0.0 \%$ & 0 & $0.0 \%$ & 0 & $0.0 \%$ & 0 & $0.0 \%$ & - \\
\hline $24 \mathrm{hr}$ & 0 & $0.0 \%$ & 0 & $0.0 \%$ & 0 & $0.0 \%$ & 0 & $0.0 \%$ & - \\
\hline $36 \mathrm{hr}$ & 0 & $0.0 \%$ & 0 & $0.0 \%$ & 0 & $0.0 \%$ & 0 & $0.0 \%$ & - \\
\hline $48 \mathrm{hr}$ & 0 & $0.0 \%$ & 0 & $0.0 \%$ & 1 & $4.0 \%$ & 0 & $0.0 \%$ & 0.387 \\
\hline
\end{tabular}

Statistically highly significant difference was found between groups in post-operative pruritus at $10 \mathrm{hr}$ and $12 \mathrm{hr}$, and significant difference between groups at $8 \mathrm{hr}$,and non-significant difference between groups at 30 min and48 from postoperative care unit (PACU) till the end of the first 48 hours after the operation (Table 12).

Table (12): Comparison between groups according to postoperative pruritus (Mean \pm SD)

\begin{tabular}{|c|c|c|c|c|c|c|c|c|c|}
\hline \multirow{2}{*}{ Pruritus Groups } & \multicolumn{2}{|c|}{ Group I } & \multicolumn{2}{c|}{ Group II } & \multicolumn{2}{c|}{ Group III } & \multicolumn{2}{c|}{ Group IV } & \multirow{2}{*}{ P-value } \\
\cline { 2 - 9 } & No. & $\mathbf{\%}$ & No. & $\mathbf{\%}$ & No. & $\mathbf{\%}$ & No. & $\mathbf{\%}$ & \\
\hline $30 \mathrm{~min}$ & 3 & $12.0 \%$ & 2 & $8.0 \%$ & 4 & $16.0 \%$ & 1 & $4.0 \%$ & 0.528 \\
\hline $1 \mathrm{hr}$ & 0 & $0.0 \%$ & 0 & $0.0 \%$ & 0 & $0.0 \%$ & 0 & $0.0 \%$ & - \\
\hline $2 \mathrm{hr}$ & 0 & $0.0 \%$ & 0 & $0.0 \%$ & 0 & $0.0 \%$ & 0 & $0.0 \%$ & - \\
\hline $4 \mathrm{hr}$ & 0 & $0.0 \%$ & 0 & $0.0 \%$ & 0 & $0.0 \%$ & 0 & $0.0 \%$ & - \\
\hline $6 \mathrm{hr}$ & 3 & $12.0 \%$ & 0 & $0.0 \%$ & 6 & $24.0 \%$ & 0 & $0.0 \%$ & 0.007 \\
\hline $8 \mathrm{hr}$ & 6 & $24.0 \%$ & 4 & $16.0 \%$ & 2 & $8.0 \%$ & 0 & $0.0 \%$ & 0.085 \\
\hline $10 \mathrm{hr}$ & 2 & $8.0 \%$ & 0 & $0.0 \%$ & 1 & $4.0 \%$ & 10 & $40.0 \%$ & $<0.001$ \\
\hline $12 \mathrm{hr}$ & 2 & $8.0 \%$ & 0 & $0.0 \%$ & 7 & $28.0 \%$ & 0 & $0.0 \%$ & $<0.001$ \\
\hline $18 \mathrm{hr}$ & 0 & $0.0 \%$ & 0 & $0.0 \%$ & 0 & $0.0 \%$ & 0 & $0.0 \%$ & - \\
\hline $24 \mathrm{hr}$ & 0 & $0.0 \%$ & 0 & $0.0 \%$ & 0 & $0.0 \%$ & 0 & $0.0 \%$ & - \\
\hline $36 \mathrm{hr}$ & 0 & $0.0 \%$ & 0 & $0.0 \%$ & 0 & $0.0 \%$ & 0 & $0.0 \%$ & - \\
\hline $48 \mathrm{hr}$ & 0 & $0.0 \%$ & 0 & $0.0 \%$ & 2 & $8.0 \%$ & 0 & $0.0 \%$ & 0.106 \\
\hline
\end{tabular}


There was a statistically significant difference between groups in postoperative pruritus at $8 \mathrm{hr}$ and nonsignificant difference between groups at
$30 \mathrm{~min} 4 \mathrm{hr} 18 \mathrm{hr} 36 \mathrm{hr}$ and48 from postoperative care unit (PACU) till the end of the first 48 hours after the operation (Table 13).

Table (13): Comparison between groups according to number of cases received piroxicam

\begin{tabular}{|c|c|c|c|c|c|c|c|c|c|}
\hline \multirow{2}{*}{$\begin{array}{l}\text { Patients } \\
\text { received Proups }\end{array}$} & \multicolumn{2}{|c|}{ Group I } & \multicolumn{2}{|c|}{ Group II } & \multicolumn{2}{c|}{ Group III } & \multicolumn{2}{c|}{ Group IV } & \multirow{2}{*}{ P-value } \\
\cline { 2 - 10 } & No. & $\%$ & No. & $\%$ & No. & $\%$ & No. & \% & \\
\hline $30 \mathrm{~min}$ & 5 & $20.0 \%$ & 2 & $8.0 \%$ & 4 & $16.0 \%$ & 1 & $4.0 \%$ & 0.285 \\
\hline $1 \mathrm{hr}$ & 0 & $0.0 \%$ & 0 & $0.0 \%$ & 0 & $0.0 \%$ & 0 & $0.0 \%$ & - \\
\hline $2 \mathrm{hr}$ & 0 & $0.0 \%$ & 0 & $0.0 \%$ & 0 & $0.0 \%$ & 0 & $0.0 \%$ & - \\
\hline $4 \mathrm{hr}$ & 3 & $12.0 \%$ & 0 & $0.0 \%$ & 2 & $8.0 \%$ & 1 & $4.0 \%$ & 0.315 \\
\hline $6 \mathrm{hr}$ & 0 & $0.0 \%$ & 0 & $0.0 \%$ & 0 & $0.0 \%$ & 0 & $0.0 \%$ & - \\
\hline $8 \mathrm{hr}$ & 4 & $16.0 \%$ & 0 & $0.0 \%$ & 2 & $8.0 \%$ & 0 & $0.0 \%$ & 0.05 \\
\hline $10 \mathrm{hr}$ & 0 & $0.0 \%$ & 0 & $0.0 \%$ & 0 & $0.0 \%$ & 0 & $0.0 \%$ & - \\
\hline $12 \mathrm{hr}$ & 0 & $0.0 \%$ & 0 & $0.0 \%$ & 0 & $0.0 \%$ & 0 & $0.0 \%$ & - \\
\hline $18 \mathrm{hr}$ & 2 & $8.0 \%$ & 1 & $4.0 \%$ & 3 & $12.0 \%$ & 0 & $0.0 \%$ & 0.315 \\
\hline $24 \mathrm{hr}$ & 0 & $0.0 \%$ & 0 & $0.0 \%$ & 0 & $0.0 \%$ & 0 & $0.0 \%$ & - \\
\hline $36 \mathrm{hr}$ & 3 & $12.0 \%$ & 2 & $8.0 \%$ & 3 & $12.0 \%$ & 1 & $4.0 \%$ & 0.719 \\
\hline $48 \mathrm{hr}$ & 1 & $4.0 \%$ & 1 & $4.0 \%$ & 1 & $4.0 \%$ & 0 & $0.0 \%$ & 0.794 \\
\hline
\end{tabular}

\section{DISCUSSION}

After any surgery rapid restoration of patient's autonomy, shortened hospital stay, decreased morbidity and costs are directly related to improved postoperative pain management. Postoperative pain is unpredictable, which explains the need for systematic prevention of pain before patient wakes up from anesthesia (Mitra et al., 2012).

This study showed that the addition of ketamine to Intravenous patient-controlled analgesia IV PCA with morphine was associated with less morphine consumed; less number of patients needed rescue analgesic, and more patient satisfaction. The occurrence of pruritus and nausea were more frequent in control group. This study observed that magnesium sulfate had no impact on morphine consumption. It showed no differences among groups in terms of pain scores, and other side effects. Murrough et al. (2013) concluded that the use of morphine infusion combined with PCA boluses may result in a better control of pain and lower morphine consumption.

NMDA receptor antagonists, such as, magnesium sulfate, and ketamine, have been previously investigated as a possible adjuvant for postoperative analgesia (Laskowski et al., 2011). Several studies have demonstrated the analgesic effectiveness of preoperatively administered ketamine during the acute postoperative period (Ding et al., 2014). A systematic review has shown the analgesic benefit of ketamine, especially in surgery that is accompanied by high levels of postoperative pain, and when combined with morphine to lower morphine consumption (Laskowsk et al., 2011). Jouguelet-Lacoste et al. (2015) demonstrated that low dose ketamine improved postoperative analgesia, reduced morphine consumption and incidence of nausea. Tan et al. (2015) demonstrated 
that an IV bolus at the beginning of surgery followed by a $24 \mathrm{~h}$ infusion decreased morphine consumption in patients undergoing total hip arthroplasty. Akhavanakbari et al. (2014) showed that adding ketamine to morphine in IV-PCA reduced pain score and morphine consumption.

In this study, ketamine has been used only for the postoperative period without pre- or perioperative administration. In line with the previous studies, we found that the morphine consumption was less in ketamine group, and the use of the low dose $(0.05 \mathrm{mg} / \mathrm{kg} / \mathrm{h})$ of ketamine was not associated with any psychotic effects. These results were also confirmed in a previous study (Hadi, 2013). Perioperative intravenous magnesium sulfate at very high doses has been reported to reduce postoperative morphine consumption but not postoperative pain scores (Albrecht et al., 2013). Murphy et al. (2013) found that the perioperative infusion of magnesium sulfate was associated with a decrease in postoperative opioid consumption; nevertheless, the decrease in opioid consumption was not associated with a decrease in opioid related side effects (eg, postoperative nausea and vomiting). In addition, they also found that perioperative magnesium sulfate infusion was associated with a decrease in visual analog scale pain scores up to 4-6 hours after surgery. Albrecht et al. (2013) reported that patients undergoing lower abdominal surgery with magnesium supplementation consumed $30 \%$ less morphine in the postoperative period compared with control patients. De Oliveira et al. (2013) found that postoperative use of magnesium sulfate reduced opioid consumption for pain after thoracotomy operations. However; this study showed less positive effect of magnesium sulfate on morphine consumption. Abdallah and Brull. (2013) compared the effects of magnesium sulfate with ketamine on postoperative analgesia and morphine consumption. They found that both drugs significantly reduced morphine consumption during the first 24 hours. In this study, the total morphine consumption was significantly lower in ketamine group, but we did not find any favorable effects of magnesium sulfate on morphine consumption. Postoperative pain scores were similar in all groups. The incidence of nausea and pruritus were more common in morphine alone group.

The major finding in the present study is a synergistic interaction between two NMDA antagonists, ketamine and magnesium sulphate. Magnesium is recognized to block calcium influx and antagonize NMDA receptor channels (Jahnen-Dechent and Ketteler, 2012), whereas ketamine binds to the phencyclidine binding site of NMDA receptors (Morgan and Curran, 2012), and modifies them via allosteric mechanisms. Because ketamine and magnesium block the NMDA receptor activation by distinct mechanisms of action, it is not surprising that a synergistic pharmacodynamics interaction between the two agents exists. Beside NMDA blocking activity, both magnesium and ketamine possess several other mechanisms of action that may be responsible for the interaction too $(\mathrm{Na}$ et al., 2011).

Ketamine interacts with calcium and sodium channels, dopamine receptors, 
cholinergic transmission, noradrenergic and serotoninergic re-uptake, together with opioid-related and anti-inflammatory effects (Hirota and Lambert, 2011).

Magnesium has been shown to reduce the activity of other presynaptic and postsynaptic calcium channels and to modulate the release of neurotransmitters. Magnesium also exhibits modulator effects on sodium and potassium currents, thus, influencing membrane potentials (Herroeder et al., 2011).

\section{CONCLUSION}

Usage of ketamine bolus dose (0.2 $\mathrm{mg} / \mathrm{kg}$ ) intravenously, followed by continuous infusion of ketamine $(0.05$ $\mathrm{mg} / \mathrm{kg} / \mathrm{h}$ ) decreased postoperative pain and analgesic consumption in the first 48 hours after surgery along with longer pain free period compared to patients who were given magnesium sulfate bolus dose (50 $\mathrm{mg} / \mathrm{kg}$ ) intravenously, followed by continuous infusion of magnesium sulfate $(10 \mathrm{mg} / \mathrm{kg} / \mathrm{h})$.

Combination of ketamine and magnesium sulfate, bolus dose of ketamine $(0.1 \mathrm{mg} / \mathrm{kg})$, and magnesium sulphate $(25 \mathrm{mg} / \mathrm{kg})$ intravenously, then continuous infusion of $(0.025 \mathrm{mg} / \mathrm{kg} / \mathrm{h})$ ketamine $+(25 \mathrm{mg} / \mathrm{kg} / \mathrm{h}) \cdot$ magnesium sulphate were better than ketamine alone or $\mathrm{mg}$ sulfate alone on postoperative total morphine consumption and patients haemodynamics.

Ketamine $(0.05 \mathrm{mg} / \mathrm{kg} / \mathrm{h})$ was safely used without any sign of toxicity. Ketamine is highly effective in postoperative pain control in major abdominal surgery without any hazards on patients. The total consumption of morphine, and additional analgesic requirements were less, while the satisfaction level of patients was higher in the ketamine group and ketamine $+\mathrm{Mg}$ Sulfate group.

\section{REFERENCES}

1. Abdallah FW and Brull R (2013): Facilitators effects of perineural dexmedetomidine on neuraxial and peripheral nerve block: a systematic review and metaanalysis. British Journal of Anesthesia. 110(6):915-25.

2. Akhavanakbari G, Mohamadian A and Entezariasl M (2014): Evaluation the effects of adding ketamine to morphine in intravenous patient controlled analgesia after orthopedic surgery. Perspect Clin Res., 5(2):85-7.

3. Albrecht E, Kirkham KR, Liu SS and Brull R (2013): Peri-operative intravenous administration of magnesium sulphate and postoperative pain: a meta-analysis. Anesthesia, 68(1):79-90.

4. De Oliveira GS, Castro-Alves LJ, Khan JH and McCarthy RJ (2013): Perioperative Systemic Magnesium to Minimize Postoperative Pain A Meta-analysis of Randomized Controlled Trials. Anesthesiology: The Journal of the American Society of Anesthesiologists, 119(1):178-90.

5. Ding $X$, Jin $S$, Niu $X$, Wang $T$, Zhao $X$, Ren H, Tong Y and Li Q (2014): Morphine with adjuvant ketamine versus higher dose of morphine alone for acute pain: a metaanalysis. International Journal of Clinical and Experimental Medicine, 7(9):2504-11.

6. Hadi BA, Daas R and Zelkó $R$ (2013): A randomized, controlled trial of a clinical pharmacist intervention in micro discectomy surgery - Low dose intravenous ketamine as an adjunct to standard therapy. Saudi Pharm J., 21(2):169-75.

7. Herroeder S, Schönherr ME, De Hert SG and Hollmann MW (2011): MagnesiumEssentials for Anesthesiologists. Anesthesiology, 114: 971-993. 
8. Hirota $K$ and Lambert DG (2011): Ketamine: new uses for an old drug? $\mathrm{Br} \mathbf{J}$ Anaesth., 107: 123-126.

9. Hurley RW and Wu CL (2010): Acute postoperative pain. Miller's Anesthesia, 7: 2757-87.

10. Jahnen-Dechent $W$ and Ketteler $M$ (2012): Magnesium basics. Clinical Kidney Journal, 5 (Suppl_1): 3-14.

11. Jouguelet-Lacoste J, La Colla L, Schilling $D$ and Chelly JE (2015): The use of intravenous infusion or single dose of lowdose ketamine for postoperative analgesia: a review of the current literature. Pain Medicine, 16(2):383-403.

12. Laskowski $K$, Stirling A, McKay WP and Lim HJ (2011): A Systematic Review of Intravenous Ketamine for postoperative analgesia. Can J Anaesth, 58(10):911-23.

13. Mitra S, Khandelwal P, Roberts K, Kumar $S$ and Vadivelu $N$ (2012): Pain relief in laparoscopic cholecystectomy - a review of the current options. Pain Practice, 12(6): 485496.

14. Morgan CJ and Curran HV (2012): Ketamine use: a review. Addiction, 107(1):27-38.

15. Murphy JD, Paskaradevan J, Eisler LL, Ouanes JP, Tomas VA, Freck EA and Wu CL (2013): Analgesic efficacy of continuous intravenous magnesium infusion as an adjuvant to morphine for postoperative analgesia: a systematic review and meta- analysis. Middle East J Anaesthesiol, 22(1):11-20.

16. Murrough JW, Iosifescu DV, Chang LC, Al Jurdi RK, Green CE, Perez AM, Iqbal S, Pillemer S, Foulkes A, Shah A and Charney DS (2013): Antidepressant efficacy of ketamine in treatment-resistant major depression: a two-site randomized controlled trial. American Journal of Psychiatry, 170(10):1134-42.

17. Na HS, Ryu JH and Do SH (2011): The role of magnesium in pain. In: Vink R, Nechifor $M$ (eds). Magnesium in the central nervous system. Pb:-University of Adelaide Press, Adelaide, South Australia, pp. 157-165.

18. Orser BA, Pennefather PS and MacDonald JF (1997): Multiple mechanisms of ketamine blockade of N-methyl-D-aspartate receptors. Anesthesiology: The Journal of the American Society of Anesthesiologists, 86(4):903-17.

19. Tan M, Law LS and Gan TJ (2015): Optimizing pain management to facilitate enhanced recovery after surgery pathways. Canadian Journal of Anesthesia, 62(2):20318. 


\section{دراسة مقارنة بين تاثير الحقن الوريدي للكيتامين أو كبريتات

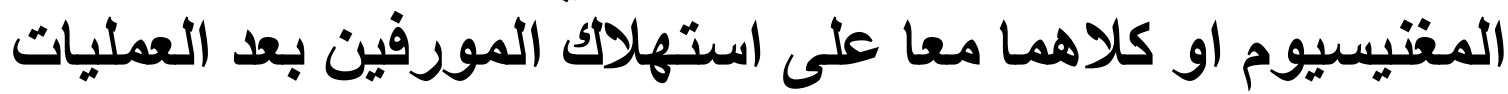

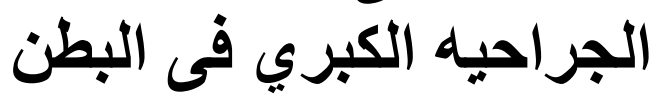

ابراهيم عبدالرؤوف محمد، طارق عبد السلام سليم موسي، عبد الوهاب عبد الستار صالح محمد

قسم التخدير والرعاية المركزة، كلية|لطب، جامعة|الأهر

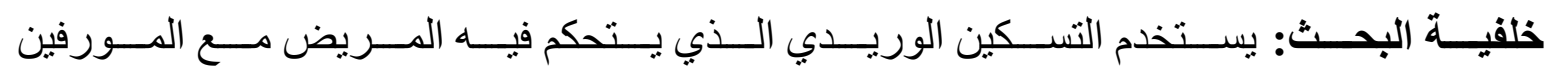

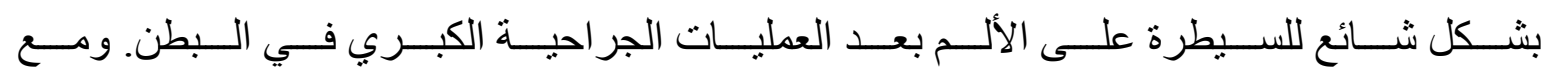

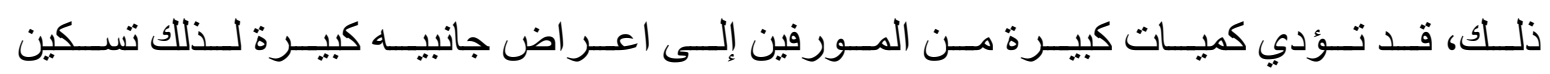

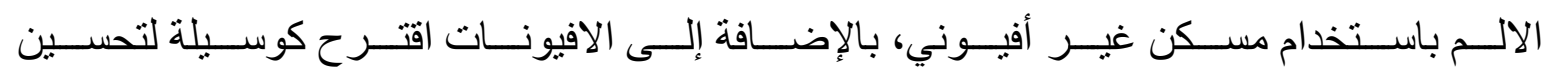
الألم بعد العملية الجراحية للعد من استخدام المو اد الأفيونية.

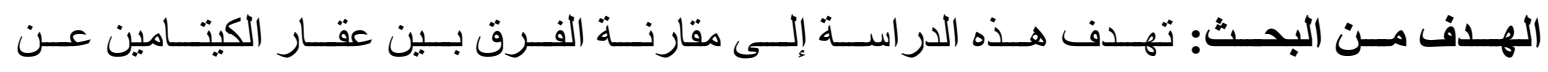

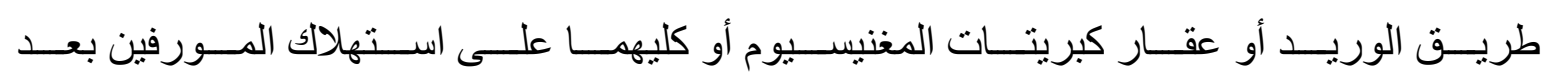
عمليات البطن الجر احيه الكبري.

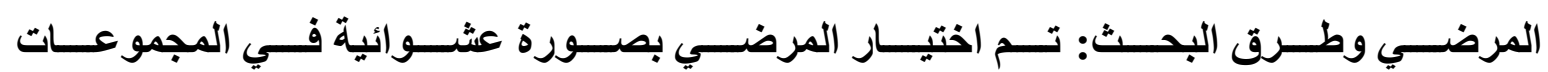

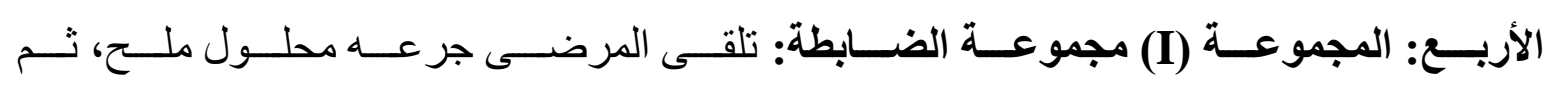

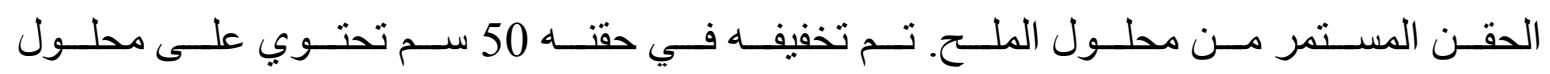

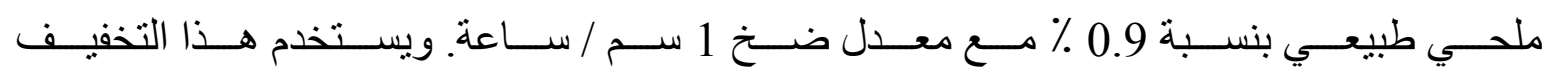

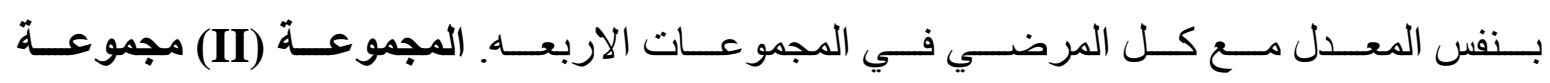

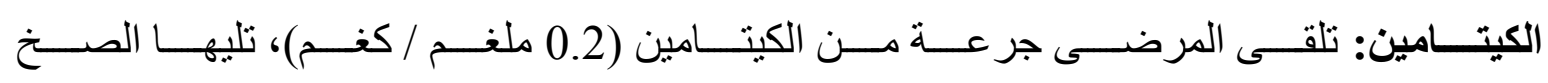

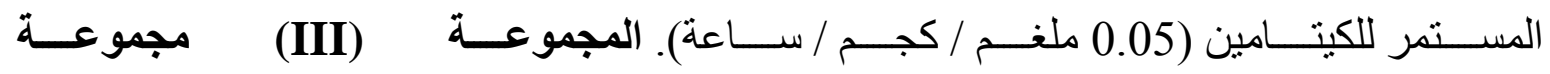

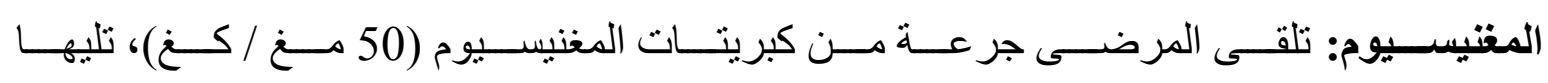

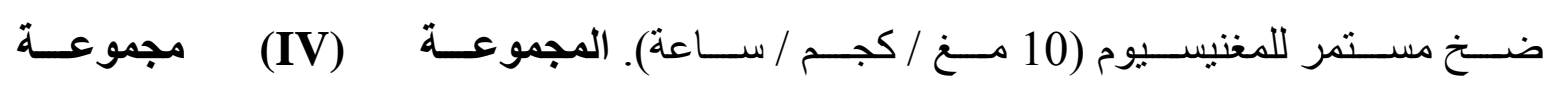

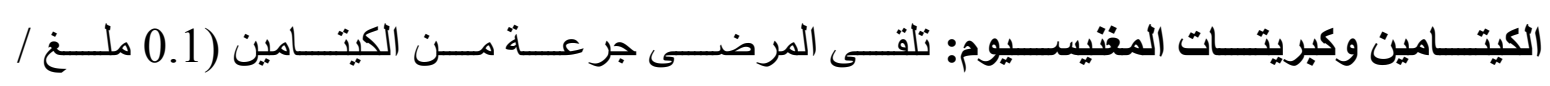

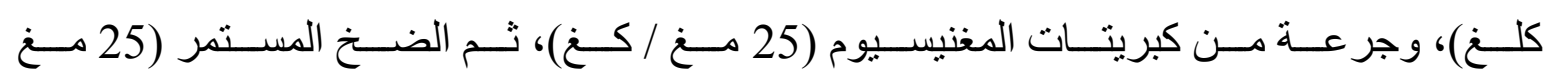

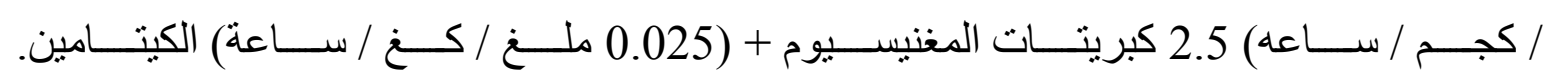




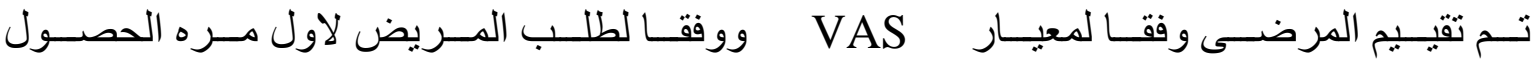

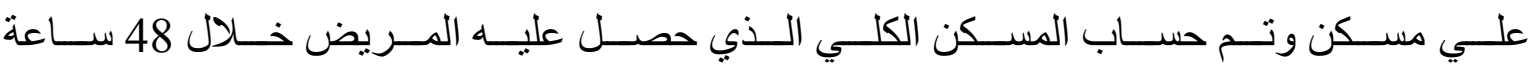

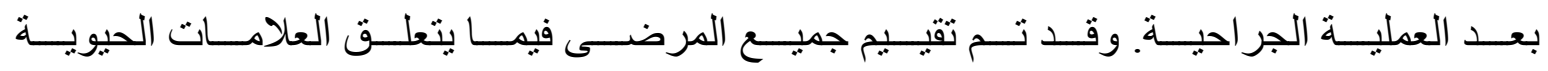

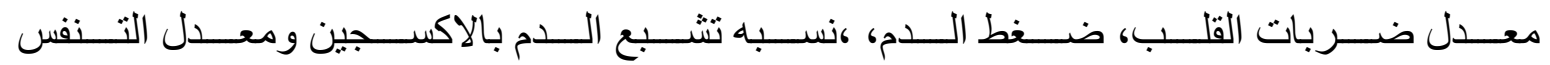

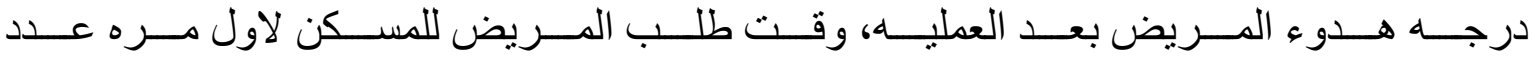

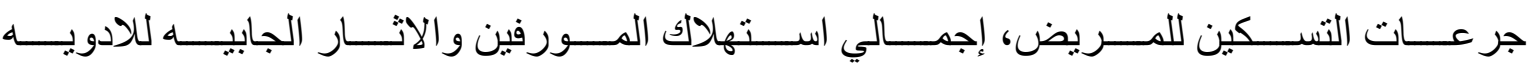
ومستوي رضا المريض.

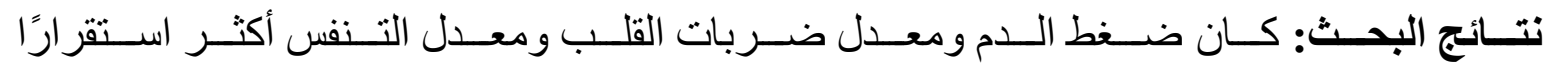

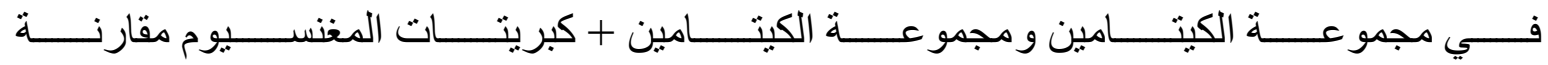

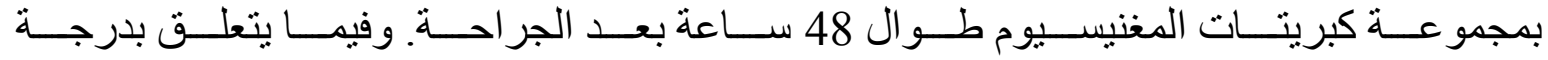

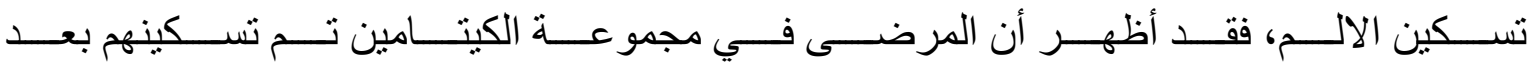

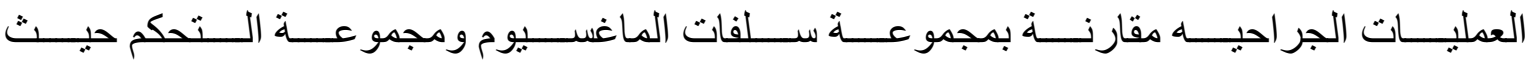

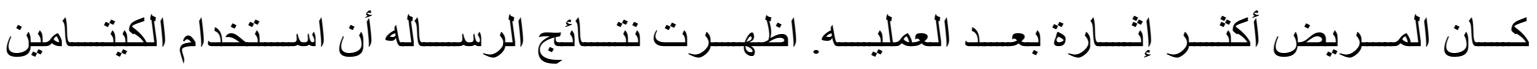

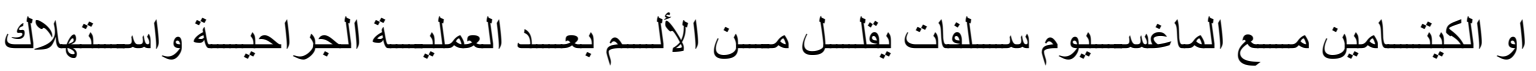

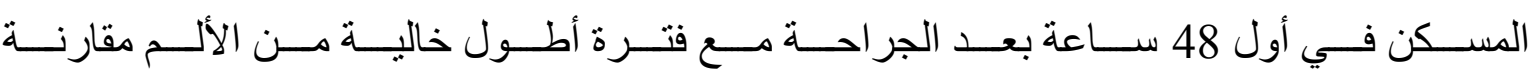
بالمرضى الذين تلقو ا كبريتات المغنيسيوم فقط.

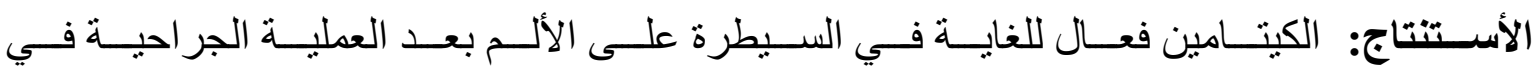

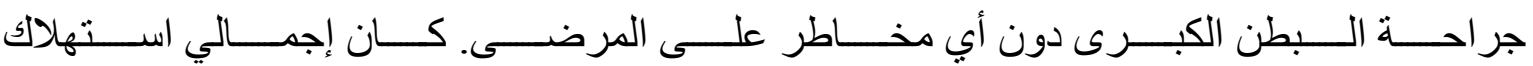

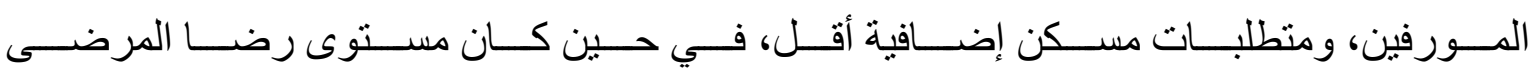
أعلى في مجمو عة الكيتامين ومجمو عه الكيتامين + كبريتات الماغنسيوم. 